\title{
21-Gene recurrence score testing among Medicare beneficiaries with breast cancer in 2010-2013
}

\author{
Julie A. Lynch, PhD, RN ${ }^{1,2}$, Brygida Berse, PhD ${ }^{1,3,4}$, Nicole Coomer, $\mathrm{PhD}^{5}$ and John Kautter, $\mathrm{PhD}^{1}$
}

Purpose: We evaluated national patient-level utilization of the 21-gene recurrence score (21-gene RS) test among Medicare beneficiaries with breast cancer. We analyzed clinical, demographic, and regional factors that predict testing.

Methods: Using 2010-2013 Medicare claims, we conducted a retrospective study of breast cancer patients. The outcome variable was whether the patient underwent testing. Independent variables expected to predict testing were age, gender, race, Medicaid status, clinical characteristics, and hospital referral region (HRR).

Results: From 2010 to 2013, the number of test orders increased by $23.0 \%$. Of the 256,818 patients identified in 2011-2012 claims, 25,352 (9.9\%) underwent the 21-gene RS test. Estrogen receptor-positive status was the strongest positive predictor of testing (odds ratio (OR) $2.58,95 \%$ confidence interval (CI) $2.48-2.69$ ). White patients were more likely to be tested than minorities (OR 1.46, 95\% CI 1.39-1.52). Secondary cancer was the strongest negative predictor. Medicaid recipients were less likely to be tested (OR 0.74, 95\% CI 0.71-0.78). The likelihood of testing decreased with increasing age and comorbidities.

Conclusions: Despite widespread implementation of the 21-gene RS test, minorities and Medicaid recipients had less access to testing. Many patients with serious comorbidities or advanced age were tested even though the risk algorithm may not have been applicable to them.

Genet Med advance online publication 23 March 2017

Key Words: breast cancer; genomic testing, 21-gene test; hospital referral region; Medicare

\section{INTRODUCTION}

Three million women in the United States live with breast cancer. One-third are Medicare beneficiaries. ${ }^{1}$ The median age of diagnosis is 61 years, and $40 \%$ of newly diagnosed cases are women over age 64 years. ${ }^{2,3}$ Approximately 96,000 Medicare beneficiaries are diagnosed yearly. The incidence rate has increased slowly, from $4.4 \%$ in 2003 to $5.4 \%$ in $2012 .{ }^{1}$ For women over age 69 , the incidence rate is expected to increase from 24 to $35 \% .{ }^{4}$ This trend has contributed to delays in care and increased costs. ${ }^{5,6}$ These dynamics illustrate the need for tools that stratify patients by risk so that clinicians can identify and prioritize patients who require aggressive treatment versus patients who may avoid or delay treatment.

One tool that stratifies patients by risk is the 21-gene recurrence score test (trade name, Oncotype DX Breast Cancer Assay) developed and conducted by Genomic Health (Redwood City, CA). The test measures tumor gene expression (16 cancer-related, including HER2, ER and $P R$, and 5 normative) to quantify the likelihood of distant recurrence at 10 years. ${ }^{7}$ Tumors are categorized as low-, moderate-, and highrisk, with an average recurrence risk of 7,14 , and $31 \%$, respectively. These risk scores are used to predict the benefit from adjuvant chemotherapy after surgery for women with earlystage, lymph node-negative (LN-), estrogen receptor-positive
(ER+), human epidermal growth factor receptor 2-negative (HER2-) breast cancer patients. ${ }^{8,9}$ The 21-gene test has been included in American Society of Clinical Oncology and National Comprehensive Cancer Network (NCCN) guidelines for treatment of stage I to IIA ER+/LN- tumors since 2007 and 2008, respectively. ${ }^{10,11}$ More recently, it has been included in NCCN guidelines for certain stage IIA-IIIB breast cancers with LN micrometastases. ${ }^{11}$ Preliminary results of the large TAILORx trial of more than 11,000 HR+/HER2-/LN- patients showed that patients with a low-risk score had remarkably low rates of recurrence with hormonal therapy alone. ${ }^{12}$ In 2016, the American Joint Committee on Cancer (AJCC) incorporated the Oncotype DX test in the Eighth Edition of the AJCC Cancer Staging Manual. ${ }^{13}$

Medicare coverage of the 21-gene test began in 2008. ${ }^{14} \mathrm{~A}$ retrospective analysis of Surveillance, Epidemiology, and End Results (SEER) data from 2005 to 2009 demonstrated that the 21-gene recurrence score influenced receipt of chemotherapy among Medicare beneficiaries. ${ }^{15}$

Several studies have analyzed the clinical utility and costeffectiveness of the 21-gene test. ${ }^{16,17}$ However, few studies have analyzed national, patient-level utilization. The aims of this study were to identify and characterize Medicare beneficiaries who underwent the test in 2011-2012, analyze factors that

${ }^{1}$ RTI International, Waltham, Massachusetts, USA; ${ }^{2}$ Department of Veterans Affairs Salt Lake City Health Care System, Salt Lake City, Utah, USA; ${ }^{3}$ Boston University School of Medicine, Boston, Massachusetts, USA; ${ }^{4}$ Veterans Health Administration, Bedford, Massachusetts, USA; ${ }^{5}$ RTI International, Research Triangle Park, North Carolina, USA. Correspondence: Julie A. Lynch (jlynch@rti.org) 
predict the likelihood to undergo testing, and evaluate concordance with guidelines.

\section{MATERIALS AND METHODS \\ Data sources}

We conducted a retrospective study using secondary data analysis methods. The primary data source was Medicare claims, including 2010-2013 100\% inpatient MedPAR, Part B, and outpatient files. Additional data sources included the denominator file (100\%), the hierarchical condition categories (HCC) risk score file (developed by RTI International), the Provider of Service file, the Health Resources and Services Administration Area Health Resource file, ${ }^{18}$ and the Dartmouth Atlas Hospital Referral Region (HRR) database. ${ }^{19}$

We used validated algorithms to identify incident breast cancer cases using Medicare claims data. ${ }^{20,21}$ We created a variable to indicate the year when a claim for breast cancer treatment was first submitted. Beneficiaries identified in 2010 claims included prevalent cases from previous years; we eliminated those patients from subsequent analysis. Beneficiaries who had initial claims in 2011 through 2013 represented newly identified cases. We analyzed and reported data for beneficiaries identified in 2011 to 2013 claims. However, our previous research revealed that approximately half the claims for the 21-gene tests were ordered more than 30 days after the surgical pathology procedure. ${ }^{22}$ Consequently, patients identified in 2013 claims may have had claims for testing in 2014 that were not available in our dataset. We therefore restricted multivariate and regional analysis to years 2011 and 2012.

Our analytic sample met the following criteria:

1. Were beneficiaries of Medicare fee-for-service who had at least one inpatient claim with a breast cancer diagnosis code (International Classification of Diseases, ninth revision (ICD-9), diagnosis codes 174.0-175.9) within MedPAR or two or more outpatient or Part B claims on separate dates with a breast cancer diagnosis code.

2. Had a short-term or specialty hospital stay in a physician office, inpatient or outpatient hospital, or ambulatory surgical center.

3. Sought breast cancer treatment in 2011-2013, defined as having a breast biopsy and/or a breast surgery (ICD-9 procedure codes 85.11-85.48) claim in MedPAR, or breast biopsy (CPT/HCPCS codes 10021-10022; 1910019103 ; 76942), surgical pathology analysis $(88305,88307$, $88309)$, or a complex diagnostic test (83890-98; 8390014; 83950-51; 86215; 86225; 86294; 86300-05; 86316; 87149; 88371-72) in outpatient or Part B files.

We restricted our analysis to claims that had breast cancer listed as the line item or principal diagnosis code. MedPAR, outpatient, and Part B claims were placed in a patient-level analytic file that became the basis for the study.

\section{Variables}

The unit of observation was the patient. The outcome variable was whether the patient had a claim for the 21-gene test. This was coded as 1 if the claim was identified as Genomic Health's Clinical Laboratory Improvement Amendment (CLIA) 05D1018272. Independent variables expected to predict testing included patient demographics (age, gender, race), Medicaid status, clinical characteristics (ER status, LN status, secondary cancer, end-stage renal disease (ESRD), HCC score, inpatient stay for breast surgery, other molecular tests), and regional characteristics. We also analyzed the correlation between undergoing the 21-gene test and undergoing a similar genomic test for breast cancer. This was identified by a claim with the CLIA number for Agendia (Irvine, CA), the supplier of the Symphony suite of microarray-based assays for clinical management of breast cancer. These include the 70-gene signature test (MammaPrint) and TargetPrint and BluePrint. MammaPrint is used for selection of breast cancer patients unlikely to benefit from adjuvant chemotherapy. ${ }^{23}$ TargetPrint provides a quantitative assessment of tumor receptor status by measuring messenger RNA expression levels of three markers (ER, $P R$, and HER2). The BluePrint test identifies the molecular subtype for breast cancer-based expression patterns of 80 genes and refines the prognostic ability of MammaPrint. In this analysis, the existence of a claim with Agendia was treated as an independent variable.

Patient demographic variables and postal codes of residence were obtained from the Medicare Denominator enrollment file. Postal code was used to characterize the region in which the patient lived (county, HRR, Urban Influence Code, and distance from a National Cancer Institute (NCI) cancer center).

Clinical characteristics obtained from claims data included ER status (V86.0-V86.1), LN status (196.0-196.9), secondary cancers (197.0-198.9), and ESRD (585.6). Secondary cancers and ESRD diagnoses were included to identify patients who were inappropriate candidates for the test. Each patient's health insurance claim number was used to obtain the weighted average HCC score, which served as a proxy to identify the patient's health status. This number is derived from a patient's prior year of claims to predict future costs. It represents the relative expenditures that are likely to be incurred for a patient, based on factors such as age, sex, Medicaid status, and individual disease groups. ${ }^{24}$ Patients with lower HCC scores are considered healthier.

Most independent variables, including the HRR, were dichotomous; others such as age and HCC score were continuous. Age was measured in years. Distance was measured in miles. HCC score was measured in increments of 0.001 .

\section{Statistical analysis}

Statistical analysis was performed using Stata software (version 12.0; StataCorp, College Station, TX). We conducted univariate and bivariate analyses and obtained descriptive statistics for all 
variables. As appropriate, group differences were tested using the chi-square test of independence for categorical variables and $t$-tests or ANOVA for continuous variables. We performed multivariate logistic regression to identify characteristics associated with a claim for the 21-gene test. We performed sensitivity analysis to ensure that the observed effects were not attributable to an artifact of modeling.

\section{RESULTS}

\section{Claims and payments}

Between 2010 and 2013, we identified 50,873 claims for the 21-gene test (Table 1). The number of claims per year increased by $23 \%$, from 10,988 in 2010 to 13,517 in 2013 . The mean Medicare payment for the test remained stable at approximately $\$ 3,311$, and the total expenditures amounted to $\$ 168,418,052$ over the 4-year period. There were fewer claims for the 70-gene signature and other tests from Agendia. However, the number of claims for Agendia tests increased more than fourfold, from 308 in 2010 to 1,356 in 2013.

We identified 383,070 patients who had a new claim for breast cancer biopsy, surgery, complex laboratory diagnostic tests, or surgical pathology procedure from 2011 to 2013 for breast cancer diagnosis or treatment. Bivariate analysis identified several demographic, clinical, and regional characteristics associated with a claim for the 21-gene test. Table 2 summarizes patient characteristics by testing status from 2011 through 2013.

\section{Demographic and clinical characteristics of patients tested} Of the 256,818 patients identified in 2011 through 2012, 25,352 (9.9\%) had a claim for the 21-gene test. Patients tested were younger than those not tested (mean $=69.2$ years, $\mathrm{SD}=6.9$ for tested; mean $=73.0, \mathrm{SD}=10.0$ for not tested). The highest percentage of patients tested (16.0\%) were 65-69 years of age. Women were more likely to be tested than men (9.9 vs. $7.5 \%$ ). Analysis of testing across race/ethnicities revealed that among non-Hispanic whites who underwent breast cancer treatment, $10.4 \%$ were tested compared to $6.8 \%$ of Hispanics and $7.0 \%$ of non-Hispanic blacks. There was a negative association between Medicaid status and testing. Only $7.4 \%$ of Medicaid recipients were tested, compared with $10.4 \%$ of those who did not receive Medicaid.

Analysis of clinical characteristics illustrated substantial undercoding of ER status, LN status, and potentially secondary cancers. These variables are needed to identify the eligible patient population. ER status was missing from claims for $68.5 \%$ of patients. $\mathrm{LN}+$ disease was coded for only $12.1 \%$ of patients, and secondary cancer was coded for $6.1 \%$ of patients; these rates were substantially lower than the prevalence of LN+ and metastatic breast cancers in cancer registries. ${ }^{25}$

The 21-gene test is specifically designed for ER+ patients. Accordingly, ER+ patients were much more likely to be tested than ER-patients (21.4 vs. $2.0 \%$ ). $\mathrm{LN}+$ patients were more likely to be tested than those who did not have a code for $\mathrm{LN}+$ disease (13.6 vs. $9.4 \%$ ). Only $2.6 \%$ of patients with a secondary cancer diagnosis were tested, which was expected because the test is recommended for early-stage breast cancer patients. Analysis of the HCC score revealed that patients with fewer comorbidities were more likely to be tested. The mean HCC score of patients tested was $0.72(\mathrm{SD}=0.56)$ compared to $0.97(\mathrm{SD}=$ 0.82 ) for patients not tested. Yet, some patients with serious conditions such as ESRD were still tested. Having an inpatient stay was negatively associated with testing. Undergoing other molecular tests was positively associated with undergoing the 21 -gene test. Patients who underwent another breast cancer genomic test supplied by Agendia were almost twice as likely to undergo the 21-gene test.

\section{Regional characteristics}

We conducted several analyses to measure the association between residence and testing status. The number and percentage of patients tested varied widely by state (as shown in Supplementary Table S1 online). New Mexico had the highest percentage (14\%) of patients tested, and Rhode Island and the Commonwealth of Puerto Rico had the lowest percentage (each at $4-5 \%$ ). We also analyzed the association between patient proximity to an NCI cancer center and testing status. In contrast to our previous research analyzing lung cancer molecular testing, ${ }^{26}$ distance to an NCI cancer center was not significant. There was also no association between testing status and county-level income or education level. There was little variability in testing between various metropolitan and nonmetropolitan areas, which were classified according to Urban Influence $\operatorname{Codes}^{27}$ (Supplementary Table S2 online).

We then considered the association between HRR and testing status. Some HRRs were positively associated with testing, whereas others were negatively associated. The distribution of

Table 1 Claims for 21-gene and 70-gene breast cancer tests among Medicare beneficiaries in 2010 and 2013

\begin{tabular}{|c|c|c|c|c|c|c|}
\hline & \multicolumn{6}{|c|}{ Year } \\
\hline & 2010 & 2011 & 2012 & 2013 & Total & Percent change \\
\hline 21-gene RS test & 10,988 & 12,976 & 13,392 & 13,517 & 50,873 & 23.0 \\
\hline Mean payments & $\$ 3,323$ & $\$ 3,302$ & $\$ 3,324$ & $\$ 3,296$ & $\$ 3,311$ & -0.8 \\
\hline Total payments & $\$ 36,508,894$ & $\$ 42,849,866$ & $\$ 44,513,830$ & $\$ 44,545,463$ & $\$ 168,418,052$ & 22.0 \\
\hline Agendia tests ${ }^{a}$ & 308 & 436 & 856 & 1,356 & 2,956 & 340.3 \\
\hline Mean payments & $\$ 3,411$ & $\$ 3,647$ & $\$ 3,014$ & $\$ 3,348$ & $\$ 3,302$ & -1.8 \\
\hline Total payments & $\$ 1,050,588$ & $\$ 1,590,092$ & $\$ 2,579,984$ & $\$ 4,539,888$ & $\$ 9,760,552$ & 332.1 \\
\hline
\end{tabular}

${ }^{a}$ Agendia breast cancer tests identified in claims include MammaPrint, TargetPrint, and BluePrint.

Data from RTI International's analysis of Medicare claims 2010 through 2013. 


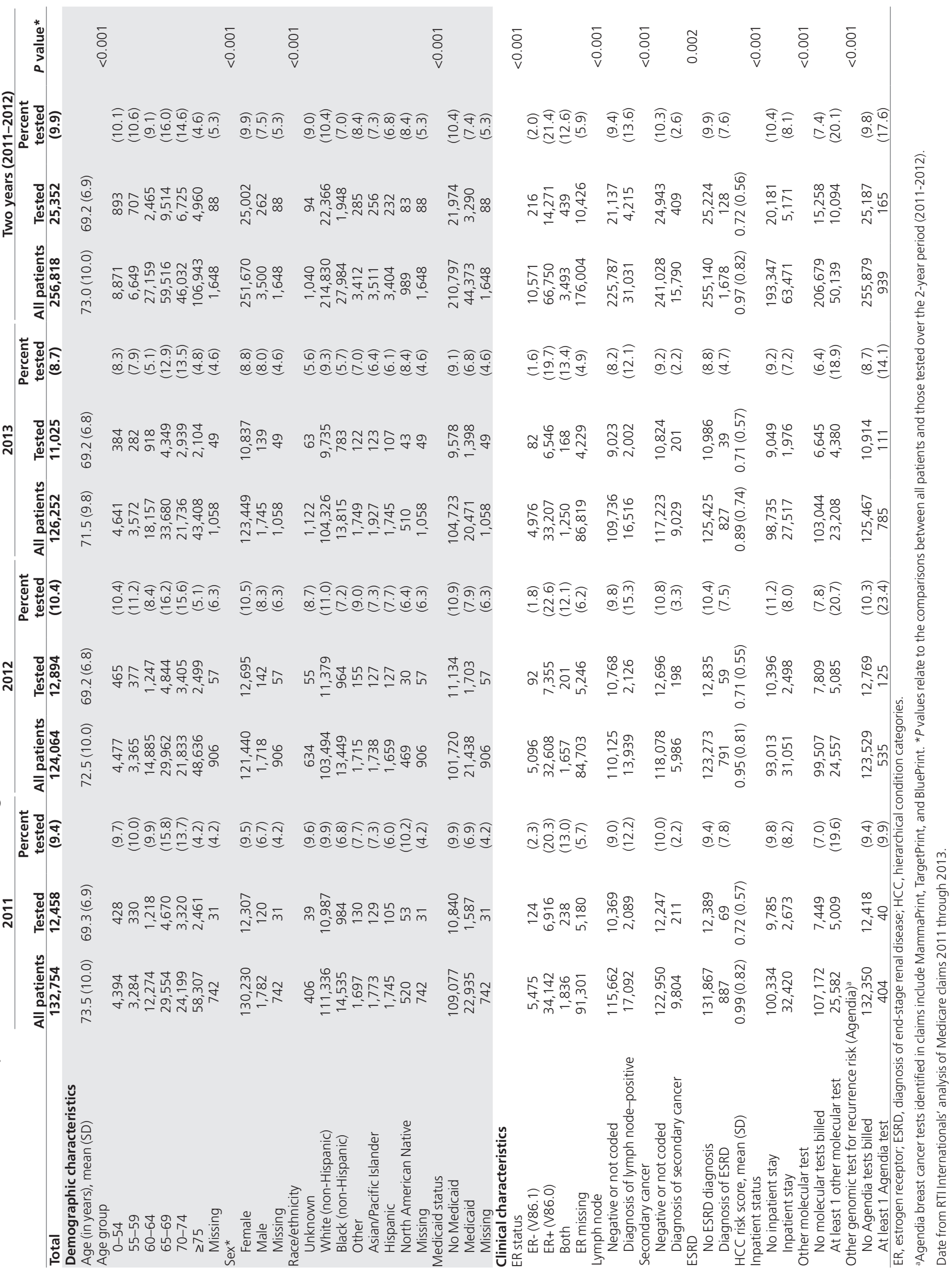


21-gene RS testing among HRRs is presented in Figure 1. Data used to create the figure are presented in Supplementary Table S3 online. There was an eightfold difference between HRRs with the highest and the lowest levels of testing, ranging from $2.9 \%$ in Ogden, Utah, to $23.6 \%$ in Terre Haute, Indiana.

\section{Prediction model}

The relationship between several independent variables and the likelihood of undergoing the 21-gene test persisted in multivariate logistic regression analysis. Table 3 summarizes the results of the logistic regression model.

Several demographic characteristics predicted testing. A yearly increase in age decreased the likelihood of testing by $4.0 \%$ (odds ratio (OR) 0.96, confidence interval (CI) 0.95-0.96). Non-Hispanic white patients were more likely to be tested (OR 1.46, CI 1.39-1.52). Medicaid recipients were less likely to be tested (OR 0.74, CI 0.71-0.78).

Several clinical characteristics positively predicted testing. Patients coded with ER+ breast cancer were almost four times as likely to undergo the 21-gene test (OR 3.90, CI 3.79-4.02). Patients who underwent another breast cancer molecular test were also more likely to undergo the 21-gene test (OR 2.80, CI 2.72-2.89). Clinical characteristics that were negative predictors of testing included secondary cancer diagnosis (OR 0.16, CI 0.14-0.17), ESRD diagnosis (OR 0.82, CI 0.67-0.99), and inpatient stay (OR 0.89 , CI $0.86-0.92$ ). Poor health status, as measured by HCC score, was also a negative predictor of testing. To interpret the OR $(0.68$, CI $0.66-0.69)$ of HCC score (which is measured in increments of 0.001), we obtained the logit coefficient. For every 0.10-point increase in HCC score, there was a $4.8 \%$ decrease in likelihood of being tested.

HRRs were both strong positive and negative predictors of testing. After adjusting for other demographic and clinical characteristics included in the model, patients most likely to be tested lived in McAllen, Texas (OR 2.84, CI 1.95-4.12). Patients who lived in Ogden, Utah, were least likely to be tested (OR 0.20 , CI 0.09 to 0.44 ). Overall, there were 54 HRRs that were positive predictors of testing and 57 HRRs that were negative predictors.

\section{DISCUSSION}

This is the first study to analyze utilization of the 21-gene test among all Medicare beneficiaries with breast cancer. Several studies have analyzed test utilization among smaller groups of patients. ${ }^{28-30}$ One study of 7,375 patients from 2006 to 2008 found disparities in access to testing among black patients and those with lower education levels. ${ }^{31}$ Two larger studies of 70,802 and 44,044 patients in the SEER registry data linked to Medicare claims found a fourfold increase in testing from 2005 through 2009 and reported little variability in testing across SEER regions or by patient demographics. ${ }^{15,32}$ Our populationlevel analysis of 256,818 Medicare patients found variations in access according to age and health status, which is consistent with clinical guidelines. However, in contrast to the study by Dinan et al. ${ }^{32}$ our study identified income, racial, and regional variations in access to the 21 -gene test. In bivariate analyses,

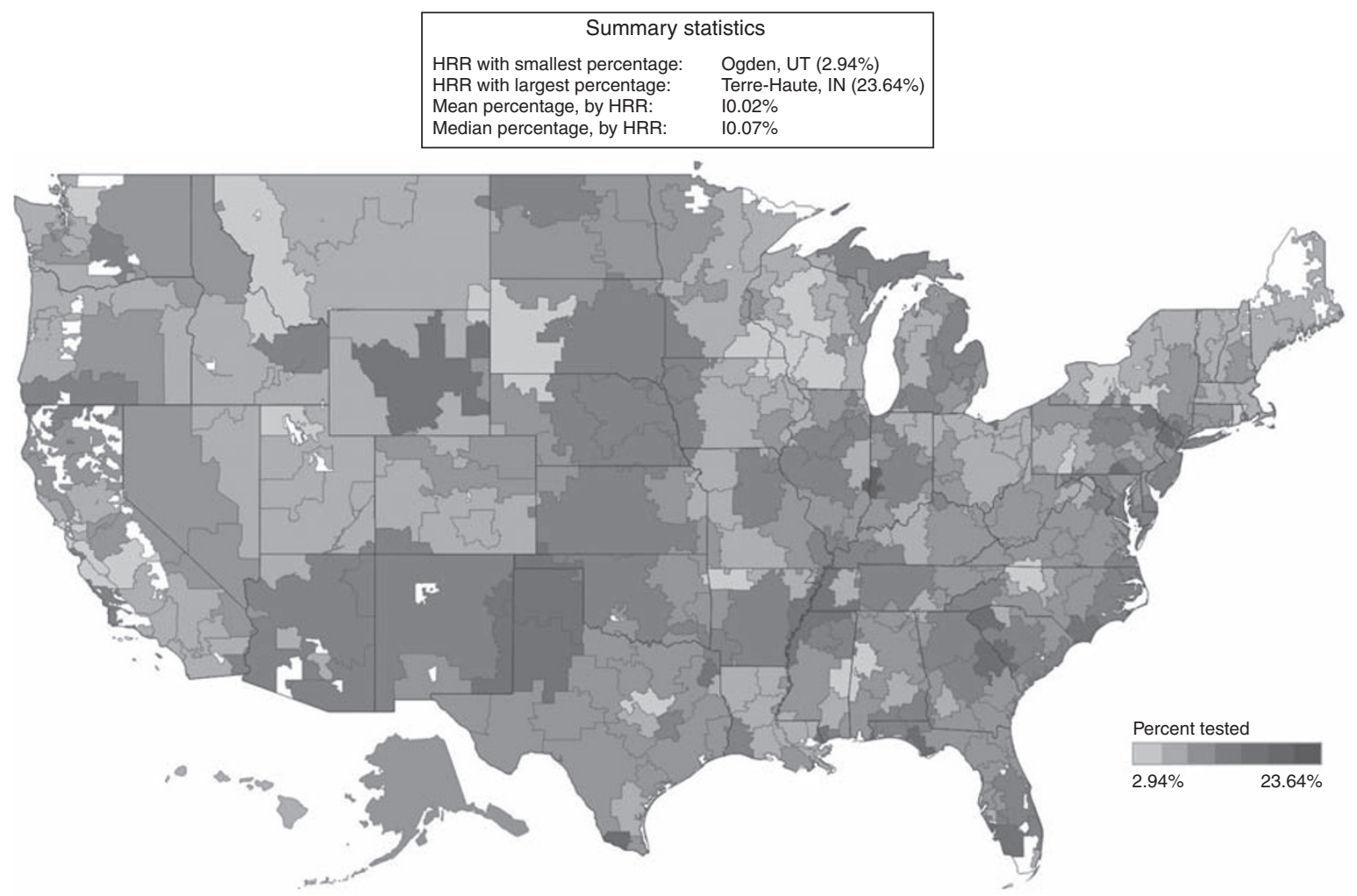

Figure 1 Distribution of 21-gene RS testing by Hospital Referral Region in 2011-2012. The denominator is the number of patients who had breast tissue analyzed. 
Table 3 Characteristics that predict use of the 21-gene RS test for breast cancer patients Independent variables Clinical and demographic characteristics

\begin{tabular}{|c|c|}
\hline & $\begin{array}{l}\text { Age (per year) } \\
\text { Non-Hispanic white (versus all other) } \\
\text { Medicaid recipient (versus nonrecipient) } \\
\text { Coded as ER+ breast cancer } \\
\text { Lymph node+ cancer } \\
\text { Coded with secondary cancer } \\
\text { ESRD diagnosis } \\
\text { Weighted average HCC score (per } 0.001 \text { increase) } \\
\text { Inpatient surgery } \\
\text { Receipt of another molecular test }\end{array}$ \\
\hline \multicolumn{2}{|l|}{ HRR: state - city } \\
\hline & TX-McAllen \\
\hline & IN - Terre Haute \\
\hline & $\mathrm{OH}$ - Elyria \\
\hline & LA - Slidell \\
\hline & NJ - Morristown \\
\hline & SC - Greenville \\
\hline & TX - Longview \\
\hline & PA - York \\
\hline & PA - Lancaster \\
\hline & LA - Lake Charles \\
\hline & GA - Augusta \\
\hline & KY - Covington \\
\hline & NY - East Long Island \\
\hline & $\mathrm{AL}-$ Dothan \\
\hline & Washington, DC \\
\hline & CA - Santa Cruz \\
\hline & MD - Takoma Park \\
\hline & NJ - Newark \\
\hline & MS - Tupelo \\
\hline & SC - Florence \\
\hline & IN - Munster \\
\hline & NM - Albuquerque \\
\hline & MO - Cape Girardeau \\
\hline & FL - Panama City \\
\hline & IL - Blue Island \\
\hline & Ml - Saginaw \\
\hline & TX-Bryan \\
\hline & NJ - Paterson \\
\hline & PA - Scranton \\
\hline & PA - Sayre \\
\hline & IN - Gary \\
\hline & TN - Memphis \\
\hline & AZ - Tucson \\
\hline & Ml - Flint \\
\hline & VA - Arlington \\
\hline & Ml-Dearborn \\
\hline & NJ - Hackensack \\
\hline & $\mathrm{Ml}$ - Ann Arbor \\
\hline & $\mathrm{OH}$ - Cincinnati \\
\hline & $\mathrm{HI}$ - Honolulu \\
\hline & TX-Amarillo \\
\hline & NJ - Ridgewood \\
\hline & $\mathrm{Ml}$ - Detroit \\
\hline & Ml- Lansing \\
\hline & NC - Wilmington \\
\hline & AL - Mobile \\
\hline & NY - New York \\
\hline & IL - Melrose Park \\
\hline
\end{tabular}

$\begin{array}{ll}\text { OR } & P \\ 0.96 \\ 1.46 \\ 0.74 \\ 3.90 \\ 0.96 \\ 0.16 \\ 0.82 \\ 0.68 \\ 0.89 \\ 2.80\end{array}$

$P$ value

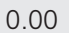

0.00

0.00

0.00

0.07

0.00

0.04

0.00

0.00

0.00

2.84

2.77

2.61

2.28

2.26

2.15

2.02

2.01

1.99

1.96

1.85

1.83

1.82

1.82

1.82

1.80

1.75

1.74

1.73

1.72

1.70

1.69

1.64

1.64

1.63

1.63

1.62

1.61

1.58

1.55

1.54

1.53

1.53

1.52

1.51

1.51

1.51

1.48

1.47

1.47

1.46

1.46

1.44

1.43

1.39

1.38

1.38

1.37
0.00

0.00

0.00

0.00

0.00

0.00

0.00

0.00

0.00

0.00

0.00

0.00

0.00

0.00

0.00

0.01

0.00

0.00

0.00

0.00

0.01

0.00

0.01

0.02

0.00

0.00

0.05

0.01

0.01

0.05

0.01

0.00

0.00

0.01

0.00

0.01

0.00

0.00

0.00

0.01

0.03

0.03

0.00

0.02

0.03

0.03

0.00

0.01
$95 \% \mathrm{Cl}$

\subsection{5}

1.39

0.71

3.79

0.93

0.14

0.67

0.66

0.86

2.72

1.95

1.81

1.74

1.39

1.77

1.68

1.31

1.44

1.48

1.28

1.39

1.25

1.52

1.30

1.49

1.18

1.29

1.35

1.22

1.22

1.17

1.34

1.11

1.07

1.26

1.25

1.00

1.12

1.11

1.01

1.13

1.23

1.19

1.12

1.20

1.09

1.18

1.16

1.16

1.11

1.04

1.03

1.15

1.05

1.03

1.03

1.13

1.08 group for HRR is Birmingham, Alabama, which had the median percentage of patients tested (10.07\%).

$\mathrm{Cl}$, confidence interval; ER, estrogen receptor; ESRD, end-stage renal disease; HCC, hierarchical condition categories; HRR, Hospital Referral Regions; OR, odds ratio. Data from RTI International's analysis of 2011-2013 Medicare claims data.

Table 1 Continued on next page 
Table 3 Continued

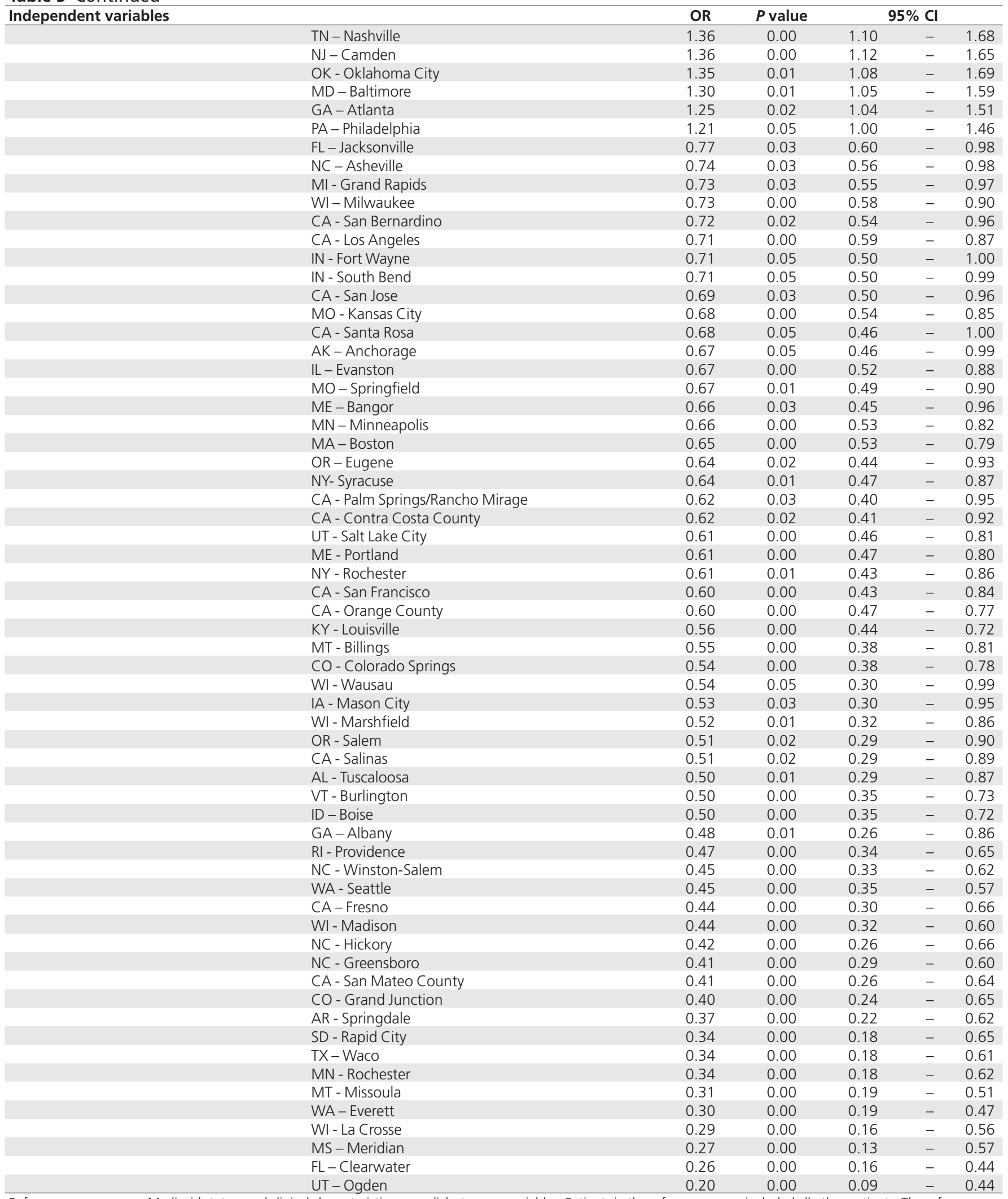

Reference groups: race, Medicaid status, and clinical characteristics were dichotomous variables. Patients in the reference group included all other patients. The reference group for HRR is Birmingham, Alabama, which had the median percentage of patients tested (10.07\%).

$\mathrm{Cl}$, confidence interval; ER, estrogen receptor; ESRD, end-stage renal disease; HCC, hierarchical condition categories; HRR, Hospital Referral Regions; OR, odds ratio. Data from RTI International's analysis of 2011-2013 Medicare claims data. 
Hispanics and blacks were tested less often than non-Hispanic whites. In multivariate analyses, non-Hispanic whites were 1.5 times more likely to be tested than non-whites. Higher rates of ER- and triple-negative breast cancer among black and Hispanic women may explain some differences. If ER status was reliably coded in the claims, then we could have analyzed this, but ER status was missing in most cases. Also, we cannot exclude that testing may have positively affected reporting of $\mathrm{ER}+$ status because the test is covered only for ER+ patients. By contrast, Medicaid status was a reliable indicator of poverty, and Medicaid recipients were much less likely to undergo testing, illustrating income disparities in access to genomic medicine.

Analysis of regional factors that predict testing demonstrate that, although there has been widespread implementation of the 21-gene test, there is also a modest level of both underutilization and inappropriate utilization. A comparison of the 57 HRRs where patients were less likely to be tested and other benchmarking measures demonstrated that many of these regions also have lower acute-care hospital capacity than the national average. Constrained capacity to care for patients probably impacts delivery of guideline-concordant cancer genomics care. Interestingly, the second lowest rate of testing occurred in Rochester, Minnesota, the location of an important medical research facility (Mayo Clinic) that also has a large commercial reference laboratory. One explanation for the low utilization of the 21-gene test in the Rochester, Minnesota, HRR may be that the Mayo Clinic was testing patients as part of its Breast Cancer Genome Guided Therapy protocol (BEAUTY). Rochester, Minnesota, also has fewer acute-care hospital beds per 1,000 residents than the national average. ${ }^{19}$

In HRRs where patients had significantly higher ORs than the national average, there may be overutilization of testing. We identified patients who were tested despite having comorbid clinical conditions that put into question the clinical utility of the test. However, our prediction model revealed that patients with secondary cancers, ESRD, and higher HCC scores were less likely to be tested. Still, utilization among these patients was not zero. Clinical guidelines and Medicare reimbursement policies specify that testing should be applied only for ER+ patients with early-stage, predominantly LN- disease for whom test results impact clinical decisions. If chemotherapy is precluded as a treatment option by a patient's comorbid conditions, age, or stated declination of chemotherapy, then the test has no clinical utility. Furthermore, the recurrence-risk algorithm may not be applicable to patients with serious comorbid medical conditions such as ESRD or those at an advanced age. The life expectancy for ESRD patients is 7.9 years. ${ }^{33}$ These patients are at greater risk for cancer and there are significant challenges in treating cancer patients with chemotherapy when they also have ESRD. ${ }^{34}$ There is no evidence that the algorithm for the 21-gene test would be valid in a patient population with extensive comorbidities.

Consistent with other studies of regional variation in healthcare delivery, our results illustrate that use of cancer genomics is subject to local practice patterns. We conclude that clinicians who order other molecular tests are also likely to order the 21-gene test. Remarkably, patients who underwent the 70-gene genomic test, which has similar utility for selecting patients for adjuvant chemotherapy, were more likely to undergo the 21-gene test.

Reliance exclusively on claims data limited this analysis in several ways. It is not feasible to use claims data to conclusively identify newly diagnosed breast cancer cases. By eliminating the 2010 claims and restricting analysis to patients who had new claims for breast surgical pathology procedures in 2011-2012, we identified a cohort of mostly newly diagnosed patients who had tissue available for testing. However, we know from SEER data that there are approximately 96,000 newly diagnosed breast cancer cases per year among Medicare beneficiaries. Clearly, therefore, our denominator included some prevalent patients who may have been previously diagnosed and tested prior to their eligibility for Medicare. Undercoding of ER status, LN status, and HER2 status substantially impeded our ability to identify the appropriate patient population for testing and made it difficult to evaluate the level of concordance with clinical practice guidelines. We can develop estimates based on analysis of SEER data suggesting that $44 \%$ of newly diagnosed patients are $\mathrm{ER}+, \mathrm{LN}$-, and HER2- and may be eligible for the 21-gene test. ${ }^{2}$ This approach suggests that approximately 126,720 patients $(44 \%$ of $(96,000 \times 3))$ may be eligible. However, if we restricted the denominator to Medicare beneficiaries based on age and comorbid conditions (selecting the patients for whom the test is most likely useful), then the eligible patient population would be much smaller. In 2011-2012 claims, we identified 148,227 patients younger than age 75 . Based on SEER estimates, 65,220 (44\%) were ER+, LN-, and HER2- patients. There were 20,304 patients younger than age 75 who had claims for the 21-gene test. If all these patients were ER+, LN-, and HER2-, then approximately $31 \%$ of guideline-recommended cases were tested. There are several reasons for believing that the market penetration of the test is even higher than $31 \%$. The estimate of the ER+, LN-, and HER2- population does not consider the number of patients who have comorbid conditions that limit the clinical utility of the test. It also does not exclude patients whose stated preference is to forgo chemotherapy regardless of their risk score.

The 21-gene test is clinically most useful for young, healthy patients with newly diagnosed breast cancer. A definitive study analyzing population-level implementation of the 21-gene test in the United States would include private-payer claims data. However, data use agreements for aggregated private-payer claims prohibit researchers from identifying providers. This restriction impedes identification of the 21-gene test in privatepayer claims. Ideally, third-party payers and researchers could use claims data to identify the population for whom guidelines recommend genomic testing.

Several studies have illustrated that the 21-gene test generates cost savings for third-party payers. ${ }^{35}$ Other studies have demonstrated that the prognostic strength of a score derived from standard pathology findings can be comparable to that derived from the 21 -gene test, ${ }^{36-38}$ especially for patients with 
high- and low-risk scores as opposed to intermediate-risk scores. The intermediate-risk category remains the most challenging for both genomics-based and conventional decision making. When the TAILORx trial is completed for all patient groups (projected for December 2017), the results may shed more light on the utility of the 21-gene test for this group of patients. ${ }^{39}$

In comparison to other genomics tests, the 21-gene test has achieved remarkable market penetration. Several factors may explain the successful translation of the test. There has been a long history of understanding the importance of tumor molecular biology for breast cancer diagnosis and treatment. Therefore, it is not surprising that breast cancer genomic tests were adopted more rapidly than lung cancer genomic tests. Early coverage and consistent inclusion of the 21-gene test across several clinical practice guidelines facilitated implementation. There are also several strong patient-advocacy groups for breast cancer that have an important role in patient education. The fact that some beneficiaries are undergoing multiple tumor gene expression tests may demonstrate consumer-based demand for testing.

Despite widespread diffusion of the 21-gene test, estimates of the eligible patient population suggest that there may also be underutilization of the test. With only 25,352 patients being tested in 2011-2012 and less access for minorities, Medicaid patients, and patients seeking care in 54 HRRs, there is clearly room for improvement. Our analysis has illustrated opportunities to expand appropriate use of genomic testing to improve risk stratification among Medicare beneficiaries diagnosed with breast cancer.

\section{SUPPLEMENTARY MATERIAL}

Supplementary material is linked to the online version of the paper at http://www.nature.com/gim

\section{ACKNOWLEDGMENTS}

We thank Merry Rabb for programming and Rob Chew for datavisualization support. This research was funded by the Centers for Medicare and Medicaid Services (contract HHSM-500-200500029I). The funder only provided data for analysis and had no involvement in the research design, analysis, or development of conclusions. J.A.L. had full access to all of the data in the study and takes responsibility for the integrity of the data and the accuracy of the data analysis.

\section{DISCLOSURE}

Genomic Health, Inc., is funding a clinical trial within the Veterans Healthcare Administration. J.A.L. is the principal investigator of the study, and B.B. worked on the study. The other authors declare no conflict of interest.

\section{REFERENCES}

1. Chronic Conditions Warehouse (CCW). Medicare chronic condition charts: Cancer trends, 2003-2012. 2014. https://www.ccwdata.org/web/guest/ medicare-charts/medicare-chronic-condition-charts/\#b2_cancer_2003_2012. Accessed 1 September 2014
2. Howlader N, Noone AM, Krapcho M, et al. SEER Cancer Statistics Review, 1975-2008. 2011; based on November 2010 SEER data submission. http://seer. cancer.gov/csr/1975_2008/. Accessed 15 April 2015.

3. Jemal A, Siegel R, Xu J, Ward E. Cancer statistics, 2010. CA Cancer J Clin 2010;60:277-300.

4. The ASCO Post. AACR 2015: U.S. Breast Cancer Cases Expected to Increase by as Much as 50\% by 2030. 2015. http://www.ascopost.com/ViewNews. aspx?nid=26587. Accessed 1 May 2015.

5. Feinstein AJ, Long J, Soulos PR, et al. Older women with localized breast cancer: costs and survival rates increased across two time periods. Health Aff (Millwood) 2015;34:592-600.

6. Bleicher RJ, Ruth K, Sigurdson ER, et al. Preoperative delays in the US Medicare population with breast cancer. J Clin Oncol 2012;30:4485-4492.

7. Paik S, Shak S, Tang G, et al. A multigene assay to predict recurrence of tamoxifen-treated, node-negative breast cancer. N Engl J Med 2004;351:28172826.

8. Dowsett M, Cuzick J, Wale C, et al. Prediction of risk of distant recurrence using the 21-gene recurrence score in node-negative and node-positive postmenopausal patients with breast cancer treated with anastrozole or tamoxifen: a TransATAC study. J Clin Onco/ 2010;28:1829-1834.

9. Biroschak JR, Schwartz GF, Palazzo JP, et al. Impact of oncotype DX on treatment decisions in ER-positive, node-negative breast cancer with histologic correlation. Breast J 2013;19:269-275.

10. Harris $L$, Fritsche $H$, Mennel $R$, et al.; American Society of Clinical Oncology. American Society of Clinical Oncology 2007 update of recommendations for the use of tumor markers in breast cancer. J Clin Oncol 2007:25:5287-5312.

11. National Comprehensive Cancer Network. Clinical Practice Guidelines in Oncology. Breast Cancer Version 2.2015. 2015; http://www.nccn.org/ professionals/physician_gls/pdf/breast.pdf. Accessed 18 March 2015.

12. Sparano JA, Gray RJ, Makower DF, et al. Prospective validation of a 21-gene expression assay in breast cancer. N Engl J Med 2015;373:2005-2014.

13. Genomic Health. Genomic health announces inclusion of Oncotype $D X ®$ in the 8th edition of American Joint Committee on Cancer (AJCC) criteria for breast cancer staging. Press release, 22 December 2016.

14. Centers for Medicare \& Medicaid Services. LCD for Oncotype DX Test - Breast Cancer Prognosis (L28287). CMS: Baltimore, MD; 2008.

15. Dinan MA, Mi X, Reed SD, Lyman GH, Curtis LH. Association between use of the 21-gene recurrence score assay and receipt of chemotherapy among Medicare beneficiaries with early-stage breast cancer, 2005-2009. JAMA Oncol 2015:1:1098-1109.

16. Reed SD, Dinan MA, Schulman KA, Lyman GH. Cost-effectiveness of the 21 -gene recurrence score assay in the context of multifactorial decision making to guide chemotherapy for early-stage breast cancer. Genet Med 2013;15:203211.

17. Hannouf MB, Xie B, Brackstone M, Zaric GS. Cost effectiveness of a 21-gene recurrence score assay versus Canadian clinical practice in post-menopausal women with early-stage estrogen or progesterone-receptor-positive, axillary lymph-node positive breast cancer. Pharmacoeconomics 2014;32:135-147.

18. Health Resources and Services Administration. Area Health Resources Files (AHRF). http://ahrf.hrsa.gov/. Accessed 16 April 2015.

19. The Dartmouth Atlas of Healthcare. 2015. http://www.dartmouthatlas.org/. Accessed 1 May 2015.

20. Nattinger AB, Laud PW, Bajorunaite R, Sparapani RA, Freeman JL. An algorithm for the use of Medicare claims data to identify women with incident breast cancer. Health Serv Res 2004;39(6 Pt 1):1733-1749.

21. Chronic Conditions Data Warehouse. CCW Chronic Conditions. 2016. https:// www.ccwdata.org/web/guest/condition-categories. Accessed 11 November 2016.

22. Kautter J, Lynch J, Coomer N, Berse B, Leahy S. Design and implementation support for the Treatment of Certain Complex Diagnostic Laboratory Tests Demonstration. Report prepared for Centers for Medicare \& Medicaid Services. 2015. https://innovation.cms.gov/Medicare-Demonstrations/Treatment-ofCertain-Complex-Diagnostic-Laboratory-Tests.html.

23. Cardoso F, van't Veer LJ, Bogaerts J, et al.; MINDACT Investigators. 70-gene signature as an aid to treatment decisions in early-stage breast cancer. $\mathrm{N}$ Engl $\mathrm{J}$ Med 2016;375:717-729.

24. Pope GC, Kautter J, Ingber MJ, Freeman S, Sekar R, Newhart C. Evaluation of the CMS-HCC Risk Adjustment Model. RTI International: Research Triangle Park, NC, 2011.

25. Howlader N, Altekruse SF, Li Cl, et al. US incidence of breast cancer subtypes defined by joint hormone receptor and HER2 status. J Natl Cancer Inst. 2014;106:dju055. 
26. Lynch JA, Khoury MJ, Borzecki A, et al. Utilization of epidermal growth factor receptor (EGFR) testing in the United States: a case study of T3 translational research. Genet Med 2013;15:630-638.

27. US Department of Agriculture. Urban Influence Codes. 2016. http://www.ers. usda.gov/data-products/urban-influence-codes.aspx. Accessed 27 October 2016.

28. Ademuyiwa FO, Miller A, O'Connor T, et al. The effects of oncotype DX recurrence scores on chemotherapy utilization in a multi-institutional breast cancer cohort. Breast Cancer Res Treat 2011;126:797-802.

29. DeFrank JT, Salz T, Reeder-Hayes K, Brewer NT. Who gets genomic testing for breast cancer recurrence risk? Public Health Genomics 2013;16: 215-222.

30. Guth AA, Fineberg S, Fei K, Franco R, Bickell NA. Utilization of oncotype DX in an inner city population: race or place? Int J Breast Cancer 2013;2013: 653805.

31. Hassett MJ, Silver SM, Hughes ME, et al. Adoption of gene expression profile testing and association with use of chemotherapy among women with breast cancer. J Clin Oncol 2012;30:2218-2226.

32. Dinan MA, Mi X, Reed SD, Hirsh BR, Lyman GH, Curtis LH. Initial trends in the use of the 21-gene recurrence score assay for patients with breast cancer in the Medicare population, 2005-2009. JAMA Oncol. 2015;1:158-166.
33. van Walraven C, Manuel DG, Knoll G. Survival trends in ESRD patients compared with the general population in the United States. Am J Kidney Dis 2014;63:491-499.

34. Eneman JD, Philips GK. Cancer management in patients with end-stage renal disease. Oncology (Williston Park) 2005;19:1199-212; discussion 1212.

35. Markopoulos C. Overview of the use of Oncotype DX(囚) as an additional treatment decision tool in early breast cancer. Expert Rev Anticancer Ther 2013;13:179-194.

36. Flanagan MB, Dabbs DJ, Brufsky AM, Beriwal S, Bhargava R. Histopathologic variables predict Oncotype DX recurrence score. Mod Pathol 2008;21:12551261.

37. Klein ME, Dabbs DJ, Shuai Y, et al. Prediction of the Oncotype DX recurrence score: use of pathology-generated equations derived by linear regression analysis. Mod Pathol 2013;26:658-664.

38. Al-Baimani KS, Petkiewicz S, Song X, Pond GR, Clemons MJ, Robertson S. Using Magee equations to predict Oncotype DX recurrence scores: A potential cost and time saving strategy? J Clin Oncol. 2014;32; abstr e22109.

39. National Cancer Institute. Hormone Therapy With or Without Combination Chemotherapy in Treating Women Who Have Undergone Surgery for NodeNegative Breast Cancer (The TAILORx Trial) NCT00310180. 2015. https:// clinicaltrials.gov/show/NCT00310180. Accessed 15 April 2015. 